\title{
Quantifying the role of nanotubes in nano:nano composite supercapacitor electrodes
}

Zheng Ling, ${ }^{1}$ Andrew Harvey, ${ }^{1}$ David McAteer, ${ }^{1}$ Ian J. Godwin, ${ }^{1}$ Beata Szydłowska, ${ }^{1}$ Aideen Griffin, ${ }^{1}$ Victor Vega, ${ }^{1}$ Yongchen Song, ${ }^{3}$ Andrés Seral-Ascaso, ${ }^{2}$ Valeria Nicolosi, ${ }^{2}$ Jonathan Coleman $^{1 *}$

${ }^{1}$ School of Physics, CRANN and AMBER Centers, Trinity College Dublin, Dublin 2, Ireland

${ }^{2}$ School of Chemistry, CRANN and AMBER Centers, Trinity College Dublin, Dublin 2, Ireland

${ }^{3}$ Key Laboratory of Ocean Energy Utilization and Energy Conservation of Ministry of Education, Dalian University of Technology, Dalian 116024, China

*colemaj@tcd.ie

Abstract: Many promising supercapacitor electrode materials have high resistivity and require conductive additives to function effectively. However, the detailed role of the additive is not understood. Here, we resolve this question by applying a quantitative model for resistancelimited supercapacitor electrodes to $\mathrm{Co}(\mathrm{OH})_{2}$-nanosheet/carbon-nanotube composites. Without nanotubes, theory predicts and experiments show that while the low-rate capacitance increases linearly with electrode thickness, the high rate capacitance decreases with thickness due to slow charging. Experiments supported by theory show nanotube addition to have two effects. First, the nanotube network effectively distributes charge, increasing the intrinsic electrode performance to the limit associated with its accessible surface area. Secondly, at high-rate, the increased electrode conductivity shifts the rate-limiting resistance from electrode to electrolyte, thus removing the thickness-dependent capacitance falloff. Furthermore, our analyses quantifies the out-of-plane conductivity of the nanotube network, identifies the cross-over from 
resistance-limited to diffusion-limited behaviour and allows full electrode modelling, facilitating rational design.

\section{INTRODUCTION}

Supercapacitors (SCs) are an important class of energy-storage devices which require a combination of high electrode-capacitance and low electrode-resistance to maximize both energy and power density. ${ }^{[1]}$ Due to their extremely high theoretical capacitance and ability to form high-surface area 2-dimensional structures, transition metal hydroxides and oxides, such as $\mathrm{Co}(\mathrm{OH})_{2}$ and $\mathrm{MnO}_{2}$, are promising electrode materials. ${ }^{[2]}$ For example single layered $\beta$ $\mathrm{Co}(\mathrm{OH})_{2}$ has demonstrated an impressive capacitance of $2028 \mathrm{~F} \mathrm{~g}^{-1}$, owning to near-complete surface exposure. ${ }^{[3]}$

However, fully utilizing transition metal hydroxides such as $\mathrm{Co}(\mathrm{OH})_{2}$, has been hindered due to their moderate electronic conductivity. Although some hydroxides, such as $\mathrm{CoOOH}$, show conductivities of $>100 \mathrm{~S} \mathrm{~m}^{-1}$, even these values are far below carbon-based electrode materials. ${ }^{[4,5]}$ Practical SCs require thick electrodes to maximize areal capacitance. As a result, the low-conductivity of the electrode material leads to large equivalent series resistance, limiting power densities. Equally problematic, their low conductivity hinders charge distribution within the electrode meaning that few transition metal hydroxides have attained their theoretical capacitance for practical electrode thicknesses or scan-rates. Therefore, it has been difficult to convert promising electrode materials into working SCs with desired high areal power densities.

Adding conductive materials, such as carbon nanotubes (CNTs) or graphene, to form nano:nano composites is a common solution to solve the problem of poor conductivity in SC electrodes and has led to significant enhancements in capacitance. ${ }^{[6]}$ This strategy has also been 
used to improve the performance of lithium ion battery electrodes and electrocatalysts. ${ }^{[7,8]}$ However, although there has been a substantial amount of work using conductive nanomaterials to enhance the SC performance of transition metal hydroxides or oxides, relatively little work has been carried out to systematically explore the mechanism behind the performance improvement. In fact, myriad questions remain unanswered: do conductive additives effect the intrinsic performance or just rate effects? What aspects of the electrical properties of the electrode are important? How do you maximize performance while minimizing additive content? Virtually no papers have addressed these materials science issues.

In this work, we use liquid exfoliated $\mathrm{Co}(\mathrm{OH})_{2}$ and $\mathrm{Co}(\mathrm{OH})_{2} / \mathrm{CNT}$ composites as model systems to investigate the underlying mechanism for improving SC performance by nanoconductor addition. Using a quantitative model for electrically-limited SC electrodes, we diagnose the problems associated with increasing the thickness of $\mathrm{Co}(\mathrm{OH})_{2}$ nanosheet electrodes. We then show that these problems can be resolved by nanotube addition, but more importantly, for the first time, explain the exact role of the nanotubes. This allows us to push $\mathrm{Co}(\mathrm{OH})_{2} / \mathrm{CNT}$ composite electrodes to their upper thickness limit, showing where ion diffusion effects become limiting. Our improved understanding of electrode performance has allowed us to design an asymmetric SC device with state of the art performance.

\section{RESULTS AND DISCUSSION.}

\section{Exfoliation and characterization of $\mathrm{Co}(\mathrm{OH})_{2}$ nanosheets}

Powdered cobalt hydroxide was exfoliated to give $\mathrm{Co}(\mathrm{OH})_{2}$ nanosheets (see Figure 1a for monolayer structure) by liquid phase exfoliation (LPE, see methods). ${ }^{[9]}$ We note that LPE has not previously been used to exfoliate $\mathrm{Co}(\mathrm{OH})_{2}$ and was chosen due to its scalability and versatility. ${ }^{[10]}$ This method involves sonicating the layered power in an aqueous sodium cholate solution, coupled with a centrifugation regime ${ }^{[11]}$ designed to retain only the thinnest 
nanosheets, and yields a dispersion of nanosheets with a pale pink color as expected for $\beta$ $\mathrm{Co}(\mathrm{OH})_{2}$ (Figure 1b). ${ }^{[12]}$

We investigated the nature of the exfoliated material using both transmission electron microscopy (TEM) and atomic force microscopy (AFM). A typical TEM image is presented in Figure 1c and shows well-defined, relatively thin nanosheets. While some nanosheets were quasi-hexagonal, many had irregular shapes. The nanosheet length (i.e. the longest dimension), L, was extracted from the TEM images and is plotted as a histogram in Figure 1d (100 counts). The lengths varied from $\sim 30 \mathrm{~nm}$ to $\sim 100 \mathrm{~nm}$ with a mean of $\langle\mathrm{L}\rangle=59 \mathrm{~nm}$. AFM characterisation yielded a similar length distribution (Figure 1e inset) and more importantly gives the number of monolayers per nanosheet (N, Figure 1e, main panel). This shows the sample to be dominated by 3 - and 4-layer nanosheets, with a long tail resulting in a mean of $\langle\mathrm{N}\rangle=4.8$ monolayers per nanosheet.

We were able to produce films of $\mathrm{Co}(\mathrm{OH})_{2}$ nanosheets very simply and controllably by vacuum filtration. A scanning electron microscope (SEM) image of such a film is given in Figure 1f and shows a highly porous, disordered network of nanosheets. We could confirm the identity of the exfoliated nanosheets via Raman spectroscopy of the filtered films, with the bulk $\mathrm{Co}(\mathrm{OH})_{2}$ powder analyzed for comparison. Both materials show vibrational modes which match well with those reported $\beta-\mathrm{Co}(\mathrm{OH})_{2}$ in literature (the scheme of Raman vibrational modes of $\mathrm{Co}(\mathrm{OH})_{2}$ can be found in Figure $\mathbf{S 1}$ in supporting information, SI). ${ }^{[13]}$ The main spectral difference between the original $\mathrm{Co}(\mathrm{OH})_{2}$ powder and nanosheets is a change in the relative intensity of the different peaks, which is probably the result of reduced size and thickness caused by exfoliation.

\section{The thickness-dependent performance of $\mathrm{Co}(\mathrm{OH})_{2}$ films}


The aim of this work is to comprehensively characterise the conductivity-related limitations of SC electrodes. We approach this by investigating the relationship between their rate-dependent performance and electrode thickness. To do this, a series of $\mathrm{Co}(\mathrm{OH})_{2}$ nanosheet films with controlled thickness were produced (see methods). To eliminate any potential impacts from additives, no binder or conductive agents were added and the films were carefully washed to remove residual surfactant. The mass loading (M/A) ranged from 0.025 to $1 \mathrm{mg} \mathrm{cm}^{-}$ ${ }^{2}$, which is broad compared with other previous studies. ${ }^{[14]}$ The film thicknesses, as measured using profilometry, varied from $t \sim 120$ to $5360 \mathrm{~nm}$. While it was possible to prepare $\mathrm{Co}(\mathrm{OH})_{2}$ films with thickness $>5360 \mathrm{~nm}$, it was not possible to transfer them from membrane to current collector due to their poor mechanical properties. We found $t$ to scale linearly with $M / A$ (i.e. $M / A=\rho_{\text {film }} t$, Figure 2a), consistent with a film density of $1990 \mathrm{~kg} \mathrm{~m}^{-3}$ and a porosity $47 \%$ (using $\left.\rho_{\mathrm{Co}(\mathrm{OH}) 2}=3.6 \mathrm{~g} \mathrm{~cm}^{-3}\right) .{ }^{[15]}$ This relatively high porosity is similar to other nanosheet networks $^{[16]}$ and will allow electrolyte insertion, thus facilitating ion transport. This is important as it means diffusion limitations should not be observed except for at high film thicknesses and high scan-rates.

Electrochemical tests were conducted to study the SC performance of the as-prepared $\mathrm{Co}(\mathrm{OH})_{2}$ films. Cyclic voltammetry $(\mathrm{CV})$ was carried out in a three-electrode system in $1 \mathrm{M}$ $\mathrm{NaOH}$ electrolyte. $\mathrm{CV}$ curves were recorded from 2 to $5000 \mathrm{mV} \mathrm{s}^{-1}$, with the current normalized to electrode mass and scan-rate and presented as specific capacitance to facilitate performance comparison. Typical CVs for the thinnest $\mathrm{Co}(\mathrm{OH})_{2}$ film (thickness, $t \sim 120 \mathrm{~nm}$ ) at various scanrates are shown in Figure $2 b$. The $\mathrm{CV}$ curves show a fast current rise, even at a scan-rate of $5000 \mathrm{mV} \mathrm{s}^{-1}$, indicating the negligible resistive limitations in such thin films. We observe a pair of peaks around $0.17 \mathrm{~V}(\mathrm{vs} \mathrm{Hg} / \mathrm{HgO})$ which correspond to the oxidation/reduction of $\mathrm{Co}(\mathrm{OH})_{2} \cdot{ }^{[4]}$ The areas enclosed by the CVs show almost no change as the scan-rate increased 
500-fold. The ultrathin film has a specific capacitance of $164 \mathrm{~F} \mathrm{~g} \mathrm{~g}^{-1}$ at $10 \mathrm{mV} \mathrm{s}^{-1}$ and it retains $76 \%(125 \mathrm{~F} / \mathrm{g})$ at $5000 \mathrm{mV} \mathrm{s}^{-1}$, confirming its good rate performance.

However, a significant problem with low-conductivity electrode materials is that such promising rate performance is not retained at high electrode thicknesses. ${ }^{[17,18]}$ Figure $2 \mathrm{c}$ shows the CVs of a $5360 \mathrm{~nm}$ thick $\mathrm{Co}(\mathrm{OH})_{2}$ film at 10,500 and $5000 \mathrm{mV} \mathrm{s}^{-1}$. This thick film displays a dramatic capacity reduction as the rate is increased. The capacitance increases much more slowly with potential, consistent with slow charging due to a higher series resistance. The specific capacitance of the thicker films drops to $97 \mathrm{~F} \mathrm{~g}^{-1}$ at $10 \mathrm{mV} \mathrm{s}^{-1}$ and $0.5 \mathrm{~F} \mathrm{~g}^{-1}$ at 5000 $\mathrm{mV} \mathrm{s}^{-1}$.

To explore the effects of thickness and scan-rate quantitatively, we plot the measured capacitance, normalized to both electrode mass (Figure 2d) and area (Figure 2e), as a function of scan-rate for a number of electrodes with different thicknesses. In Figure $2 d$, the degradation of specific capacitance with increased thickness and scan-rate can clearly be observed. Strikingly, the areal capacitance at high rates actually decreases as electrode thickness is increased (Figure 2e). This is a significant problem if the aim is to maximize capacitance by increasing electrode thickness.

Empirically, at high rate, we observe the capacitance to scale inversely with scan-rate $\left(\dot{V}^{-1}\right)$, inconsistent with the $\dot{V}^{-1 / 2}$ dependence expected for diffusion limitations via the Cottrell equation. ${ }^{[19]}$ However, we have previously shown that such behavior is the hallmark of SC electrodes where the charging time is limited by the electrode resistance. ${ }^{[14,20]}$ We note that such resistance limitations can be present even for conductive materials (e.g. PEDOT PSS, $\left.\sigma \sim 10^{5} \mathrm{~S} \mathrm{~m}^{-1}\right) .{ }^{[21]}$ These resistance limitations are the main barrier for efficient utilization of low-conductivity electrode materials, even though they have high theoretical capacitances. ${ }^{[17}$, ${ }^{22]}$ We measured the in-plane conductivity of dry $\mathrm{Co}(\mathrm{OH})_{2}$ films to be $\sim 5 \times 10^{-6} \mathrm{~S} \mathrm{~m}^{-1}$ with the 
dry out-of-plane conductivity expected to be significantly lower. ${ }^{[23]}$ However, we note that some hydroxides/oxides such as $\mathrm{CoOOH}$ are known to display enhanced conductivity in the presence of electrolytes. ${ }^{[24]}$ As a result, the effective conductivity of our SC electrodes (in the presence of electrolyte) is probably somewhat higher than these dry values.

By analyzing the effect of a series resistance on charging and discharging of a capacitor, we recently showed that the specific capacitance of an electrically limited SC electrode is given by: ${ }^{[21]}$

$$
\frac{C}{M}=C_{M}\left[1-\frac{\dot{V} \tau}{\Delta V}\left(1-e^{-\Delta V / \dot{V} \tau}\right)\right]
$$

Here, $\dot{V}$ is the scan-rate, $\Delta V$ is the voltage window, $\tau$ is the time constant of the R-C circuit used to model the capacitor electrode, $C / M$ is the measured, rate-dependent, specific capacitance while $C_{M}$ is the intrinsic, rate-independent specific capacitance of the electrode material (N.B. $C$ / $M \rightarrow C_{M}$ as $\dot{V} \rightarrow 0$ ). This equation can also be applied to areal capacitance ( $C / A$ ) and volumetric capacitance $(C / V)$ by noting that $C / V=\rho_{\text {film }} C / M$ and $C / A=t \times C / V$ where $\rho_{\text {film }}$ and $t$ are electrode density and thickness. Here we differentiate between measured capacitances, which we denote by ratios (e.g. $C / M$ ) and intrinsic (or semiintrinsic e.g. $C_{A}$ ) quantities which we denote using a subscript (e.g $C_{M}$, N.B $C_{V}=\rho_{\text {film }} C_{M}$ and $\left.C_{A}=t \times C_{V}\right)$. We note that while equation 1 was originally derived for double layer capacitors, it is also approximately true for pseudocapacitors (see SI).

We used Equation (1) to fit the rate-dependent capacitance data (see Figure 2d-e), finding good fits in all cases. This is further evidence of the resistance-limited nature of these electrodes. From the fit, for each film thickness, we extract $C_{M}$ (or $C_{A}$ ) and $\tau$. Because of it's 
linearity with thickness (or $M / A$ ) (Figure 2f), $C_{A}$ illustrates the benefits of maximising electrode loading. This graph shows the expected linearity, with the thickest film displaying $C_{A}=0.2 \mathrm{~F}$ $\mathrm{cm}^{-2}$. The slope of this graph is consistent with intrinsic specific and volumetric capacities of $C_{M}=178 \mathrm{~F} \mathrm{~g} \mathrm{~g}^{-1}$ and $C_{V}=316 \mathrm{~F} \mathrm{~cm}^{-3}$. These values are relatively low for $\mathrm{Co}(\mathrm{OH})_{2}$, partially due to the fact that our nanosheets are not monolayers but also because the low electrode conductivity may in fact limit the intrinsic capacitive properties as well as the rate performance. Shown in Figure $2 \mathrm{~g}$ is the electrode time constant plotted versus $M / A$. This graph shows $\tau$ to increase from $0.03 \mathrm{~s}$ to $20 \mathrm{~s}$ over the investigated thickness range. Empirically, this increase is quadratic in $M / A$ which is an excellent example of a disadvantage of increasing capacitance by increasing electrode thickness. The increased electrode thickness results in enlarged series resistance and so amplified $\mathrm{R}-\mathrm{C}$ time constant. Large time constants result in slow charging and discharging and so reduced effective capacitance, especially at high rates.

The time constant can be modelled as the product of the capacitance and the equivalent series resistance, with the latter parameter having contributions from both electrode and electrolyte resistances. This can be written as:

$$
\tau=C\left(R_{\text {electrolyte }}+R_{\text {electrode }}\right)=C_{V} A t\left[\frac{1}{G_{A} A}+\frac{t}{\sigma_{+} A}\right]=\frac{C_{A}}{G_{A}}+\frac{C_{A}}{\sigma_{+}} t
$$

where we use $C_{A}=t \times C_{V}$ and $A$ is the electrode area, $G_{A}$ is the conductance per unit area associated with the electrolyte and $\sigma_{+}$is the out of plane conductivity of the electrode. This model correctly predicts the empirically observed quadratic scaling of $\tau$ with $M / A$ (Figure $2 \mathrm{~g}$, N.B. $\left.M / A=\rho_{\text {film }} \times t\right)$. This model also predicts a linear scaling of $\tau / C_{A}$ with $t$ which is demonstrated in Figure 2h. Fitting the data in Figure $2 \mathrm{~h}$ to Equation (2) gives an electrolyte conductance per unit area of about $\mathrm{G}_{\mathrm{A}}=3600 \pm 1800 \mathrm{~S} \mathrm{~m}^{-2}$. Taking the working electrode to counter electrode distance to be $\sim 1 \mathrm{~cm}$ allows us to estimate the electrolyte conductivity to be 
$\sim 36 \mathrm{~S} / \mathrm{m}$, close to the expected value of $20 \mathrm{~S} \mathrm{~m}^{-1}$. More interestingly, the fit gives a value of the out of plane conductivity of the $\mathrm{Co}(\mathrm{OH})_{2}$ network (in the presence of electrolyte) to be $(4.8 \pm 0.8) \times 10^{-4} \mathrm{~S} \mathrm{~m}^{-1}$. This value is much higher than the dry, in-plane value noted above $\left(\sim 5 \times 10^{-6} \mathrm{~S} \mathrm{~m}^{-1}\right)$. This may be due to partial oxidation of the $\mathrm{Co}(\mathrm{OH})_{2}$ to $\mathrm{CoOOH}$, a material which is considerably more conductive (in plane conductivity $\sim 500 \mathrm{~S} \mathrm{~m}^{-1}$ ). ${ }^{[4]}$

Access to Equations (1) and (2) allow us to consider the limitations of low-conductivity electrode materials which result in high- $\tau$ electrodes. By expanding Equation (1) to second order, it can easily be shown that, at high rate, the measured areal capacitance is given by

$$
\left(\frac{C}{A}\right)_{\text {high-rate }}=\frac{C_{A} \Delta V}{2 \dot{V} \tau}
$$

This equation clearly shows that, at high rate, the capacity retention, $(C / A)_{\text {high-rate }} / C_{A}$, scales inversely with $\tau$. This underlines the importance of ensuring as low as possible a value of $\tau$, by increasing the electrode conductivity as much as possible. To maximize the absolute capacitance at high rate, $C_{A} / \tau$ must be maximized. Combining Equations (2) and (3), we can show that:

$$
\left(\frac{C}{A}\right)_{\text {high-rate }}=\frac{\Delta V}{2 \dot{V}}\left(\frac{1}{G_{A}}+\frac{t}{\sigma_{+}}\right)^{-1}
$$

This equation explains the observation that increasing $t$ actually decreases the high-rate capacitance. This paradoxical and unwanted effect will occur so long as the electrode resistance is the rate-limiting parameter. However, in this respect, Equation (4) offers a solution: thickness increases will cease to be detrimental once the electrolyte resistance becomes rate-limiting i.e. when $\sigma_{+}>G_{A} t$. Above this point, the high-rate areal capacitance will become limited only by the electrolyte areal conductance: $(C / A)_{\text {high-rate }} \approx \Delta V G_{A} / 2 \dot{V}$ when $\sigma_{+}>>G_{A} t$. This clearly 
outlines the importance of maximizing the conductivity, especially for thick electrodes, for example by adding nanotubes.

From the above discussion, it is clear that SC electrodes based on liquid exfoliated $\mathrm{Co}(\mathrm{OH})_{2}$ nanosheets are limited in three ways. Firstly, increasing the electrode thickness yields in a quadratic increase in $\tau$, resulting in increasingly poor performance at high rates. Secondly, the intrinsic capacitance is relatively small $\left(\left\langle C_{M}\right\rangle=178 \mathrm{~F} \mathrm{~g}^{-1}\right)$ compared to chemically produced, monolayer-rich samples $\left(2028 \mathrm{~F} \mathrm{~g}^{-1}\right){ }^{[3]}$ This difference is more than can be explained by the fact that our nanosheets are $\sim 5$ layers thick, implying a factor other than surface area to be at work. Thirdly, the poor mechanical properties of nanosheet networks result in the fragmentation of $\mathrm{Co}(\mathrm{OH})_{2}$ films with thickness beyond $5 \mu \mathrm{m}$, ruling out the possibility of improving (low-rate) performance through further increases in thickness.

Such problems are generic to low conductivity, particulate electrodes and will have to be resolved before electrodes of $\mathrm{Co}(\mathrm{OH})_{2}$, or indeed any other, nanosheets can be optimized. In the past, the standard solution has been to add nanotubes or graphene to enhance the electrical properties of the electrode, ${ }^{[25]}$ or to use a nickel foam support. ${ }^{[4]}$ However, manuscripts following such strategies generally report performance enhancements rather than exploring the physical nature of the improvements. We argue that by applying Equations (1) and (2), it is possible to gain an insight into the mechanisms behind the performance increases associated with nanotube addition.

\section{Performance of $\mathrm{Co}(\mathrm{OH})_{2} / \mathrm{SWNT}$ composite electrodes}

Previous work has indicated that adding CNTs to nanosheet-based electrochemical electrodes is an efficient way to improve electrode performance. ${ }^{[22,25,26]}$ We believe the CNTs play various roles: increasing the electrical conductivity to improve charge distribution ${ }^{[8,14]}$ 
within the electrode and enhancing the mechanical properties, resulting in greater stability ${ }^{[7]}$ and achievable thickness. ${ }^{[14]}$ Additionally, SWNTs can act as spacers preventing the restacking of the $2 \mathrm{D}$ materials and facilitating the access of electrolyte. ${ }^{[27]}$ However, in depth quantitative analysis on the effect of nanotubes on nanosheet based SC electrodes has not been performed.

To investigate the effect of nanotubes on the performance of $\mathrm{Co}(\mathrm{OH})_{2}$-based SC electrodes, composite films with fixed $\mathrm{Co}(\mathrm{OH})_{2}$ loading $\left(\mathrm{M} / \mathrm{A}=0.2 \mathrm{mg} \mathrm{cm}^{-2}\right)$ but various SWNT mass fractions $\left(M_{f}=M_{\mathrm{NT}} /\left(M_{\mathrm{NT}}+M_{\mathrm{Co}(\mathrm{OH}) 2}\right)=0\right.$ to $\left.40 \mathrm{wt} \%\right)$ were prepared by the same filtration method. The composite thickness increased roughly linearly with nanotube $M_{f}$ (Figure 3a). For each sample, the film density was calculated from the thickness and total composite mass $\left(\rho_{\text {film }}=(M / A)_{\text {Comp }} / t\right)$ and was found to vary in the range $1500-2230 \mathrm{~kg} / \mathrm{m}^{3}$ (Figure 3a lower inset). This density could be used to find the volume fraction $\left(\phi=M_{f} \rho_{\text {film }} / \rho_{N T}\right)$ as shown in Figure 3a upper inset. Interestingly, the nanotube volume- and mass-fractions were approximately equal due to the similarity between film densities and nanotube density $\left(1800 \mathrm{~kg} \mathrm{~m}^{-3}\right)$.

The dry (i.e. no electrolyte), in-plane composite conductivity, $\sigma_{=}$, was measured and is plotted as a function of $M_{f}$ in Figure $3 \mathrm{~b}$. The conductivity increases from $5 \times 10^{-6} \mathrm{~S} \mathrm{~m}^{-1}$ for the pure $\mathrm{Co}(\mathrm{OH})_{2}$ film to $260 \mathrm{~S} \mathrm{~m}^{-1}$ at $1 \mathrm{wt} \%$ to $4 \times 10^{4} \mathrm{~S} \mathrm{~m}^{-1}$ at $20 \mathrm{wt} \%$. Similar to $\mathrm{MoS}_{2} / \mathrm{SWNTs}$ and $\mathrm{MnO}_{2} / \mathrm{SWNTs}^{\left[14,{ }^{28]}\right.}$ this data is consistent with percolation theory, which predicts that, above a critical volume fraction, $\phi_{c}{ }^{[29]}$ the conductivity scales with $\phi$ as:

$\sigma_{=}=\sigma_{=, 0}\left(\phi-\phi_{=, c}\right)^{n_{=}}$

where $\sigma_{=, 0}$ is related to the nanotube conductivity and $n_{=}$is the in-plane percolation exponent. ${ }^{[30]}$ Fitting our $\mathrm{Co}(\mathrm{OH})_{2} / \mathrm{SWNTs}$ composite conductivity data (Figure 3b, inset) gives a low critical 
threshold $\left(\phi_{=, c}=0.25 \pm 0.08 \mathrm{vol} \%\right)$, an exponent $(1.45 \pm 0.13)$ which is close to the value of 1.3 expected for transport in 2 dimensions as well as giving $\sigma_{=, 0}=(6 \pm 1) \times 10^{5} \mathrm{~S} \mathrm{~m}^{-1}$.

To test the effect of nanotube content on SC performance, cyclic voltammetry was performed on all composites, as well as the $\mathrm{Co}(\mathrm{OH})_{2}$ - and SWNT-only films. The SWNT films showed ideal capacitive responses with specific capacitance of $\sim 50-100 \mathrm{~F} \mathrm{~g} \mathrm{~g}^{-1}$, virtually independent of thicknesses and scan-rates (see Figure S2 in SI). ${ }^{[14]}$ Figure 3c shows the CV curves of three films with different SWNT contents at a scan-rate of $500 \mathrm{mV} \mathrm{s}^{-1}$. The specific capacitance significantly increases as the SWNT content rises. These results are consistent the nanotubes increasing the electrode conductivity via the formation of conductive networks, facilitating charge distribution and accelerating charging.

The specific capacitance (normalized to total composite mass, $(C / M)_{C o m p}$ ) of a subset of composites is plotted as a function of scan-rate in Figure 3d. This data shows an increase in capacitance at all scan-rates as $M_{f}$ is increased. For each composite, we fitted the rate-dependent data to Equation (1), extracting $\left(C_{M}\right)_{C o m p}$ and $\tau$ as fit parameters. Shown in Figure $3 \mathrm{e}$ is $\left(C_{M}\right)_{\text {Comp }}$ plotted versus $M_{f}$. The value of $\left(C_{M}\right)_{C o m p}$ stays close to the $\mathrm{Co}(\mathrm{OH})_{2}$-only value until $M_{f}$ reaches $\sim 1 \mathrm{wt} \%$ where it undergoes a rapid rise, reaching $\sim 450 \mathrm{~F} \mathrm{~g}^{-1}$ by $3 \mathrm{wt} \%$, after which $\left(C_{M}\right)_{\text {Comp }}$ falls linearly with $M_{f}$.

We can understand this behavior as follows. For nanotubes to influence the capacitance, they must be able to carry charge from the current collector to sites throughout the electrode. This predominately involves out-of-plane transport. This means we expect no capacitance increases until an out-of-plane nanotube network has formed. Figure. 3e suggests this to occur at $\sim 1 \mathrm{wt} \%$, beyond which $\left(C_{M}\right)_{\text {Comp }}$ rises rapidly as the network becomes more extensive and delivers charge to more and more of the electrode. Once $M_{f}$ reaches $\sim 3 \mathrm{wt} \%$, the nanosheet network is extensive enough to effectively deliver charge to the entire electrode and adding 
more nanotubes results in a reduction in capacitance as $\mathrm{Co}(\mathrm{OH})_{2}$ is replaced by lowercapacitance nanotubes. This final portion can be modelled by the rule of mixtures (RoM) with fitting implying intrinsic specific capacitances of $\mathrm{Co}(\mathrm{OH})_{2}$ and SWNT of 460 and $\sim 100 \mathrm{~F} \mathrm{~g}^{-1}$. This former value represents the maximum possible value for our material when all electrical limitations have been removed.

This data can be viewed another way by normalizing $C_{M}$ to the $\mathrm{Co}(\mathrm{OH})_{2}$ mass rather than the composite mass (after subtracting the nanotube contribution) to give $\left(\mathrm{C}_{M}\right)_{\mathrm{Co}(\mathrm{OH}) 2}$. This parameter represents the specific contribution of the nanosheets only and is plotted versus SWNT volume fraction in Figure 3f. At low $\phi,\left(\mathrm{C}_{M}\right)_{\mathrm{Co}(\mathrm{OH})_{2}}$ is unchanged from that measured for $\mathrm{Co}(\mathrm{OH})_{2}$. However, between $\phi=0.01$ and $0.03,\left(\mathrm{C}_{M}\right)_{\mathrm{Co}(\mathrm{OH})_{2}}$ increases rapidly before saturating at $\sim 400-450 \mathrm{~F} \mathrm{~g}^{-1}$ with this terminal value representing the ideal value of our $\mathrm{Co}(\mathrm{OH})_{2}$ in the absence of resistance limitations.

Interestingly, if we take the value of $2028 \mathrm{~F} \mathrm{~g}^{-1}$, measured by Gao for a monolayer-only sample, ${ }^{[3]}$ as the maximum achievable value for $\mathrm{Co}(\mathrm{OH})_{2}$, and divide it by the average nanosheet thickness in this work $(\langle N\rangle=4.8$, figure $1 \mathrm{e})$, we find a value of $423 \mathrm{~F} \mathrm{~g} \mathrm{~g}^{-1}$, very close to the limiting value of $\left(\mathrm{C}_{\mathrm{M}}\right)_{\mathrm{Co}(\mathrm{OH}) 2}$ shown in figure $3 \mathrm{f}$. This implies that adding nanotubes at loading level $>3 \mathrm{wt} \%$ results in the maximum possible performance of the material, limited by its accessible surface area which is of course controlled by nanosheet thickness.

That composites with $M_{f}>3 \mathrm{wt} \%$ display specific capacitances close to those expected for nanosheets with $\langle N\rangle=4.8$, indicates that film formation does not result in much loss of accessible surface area compared to that associated with dispersed nanosheets. This in turn suggests that significant restacking has not occurred during film formation. This may imply that the nanotubes are acting as spacers which prevent nanosheet restacking, maximizing access of the electrolyte to the nanosheet surface in a manner similar to that described by Gogotsi et 
al for graphene-nanoparticle systems. ${ }^{[31]}$ This mechanism would facilitate maximization of capacitance in a manner which is complementary to the improved electrical transport due to the nanotube network.

The region of rapid increase between 0.01 and 0.03 vol\% represents the growth of the SWNT network as described above. As such, it should be described by a percolation-like scaling law. Although we do not have enough data points to get a proper fit, it is clear from the figure that the data is consistent with

$$
\left(C_{M}\right)_{\mathrm{Co}(\mathrm{OH}) 2}=\left(C_{M}\right)_{0}+\left(C_{M}\right)_{\mathrm{P}}\left(\phi-\phi_{c, c}\right)^{n_{c}}
$$

where $\left(C_{M}\right)_{0}$ represents the $\mathrm{Co}(\mathrm{OH})_{2}$-only baseline value, $\left(C_{M}\right)_{P}$ is a constant, while $\phi_{c, c}$ and $n_{c}$ are the capacitive percolation threshold and exponent respectively. Interestingly, the data is consistent with $\phi_{c, c}=0.01$, unambiguously bigger than the in-plane electrical percolation threshold (0.003). This implies that capacitance percolation is not simply proportional to inplane electrical percolation. The data is consistent with a percolation exponent of $n_{c}=0.5$, which is consistent with previous data. ${ }^{[7,8,14]}$

Shown in Figure $3 \mathrm{~g}$ are values of $\tau$, extracted from fits to Equation (1), plotted versus $M_{f}$. For $0<M_{f}<1 \mathrm{wt} \%$, we see considerable scatter which we put down to experimental error. However, for $M_{f}>1 \mathrm{wt} \%$, we see a clear decay in $\tau$ from the $\mathrm{Co}(\mathrm{OH})_{2}$-only value of $\sim 1 \mathrm{~s}$ to $<0.2$ s. We associate this with the reduction of series resistance as the nanotubes enhance the outof-plane electrode resistance.

We can model this using Equation (2), by replacing $\sigma_{+}$by an equation which represents the $\phi$-dependence of the out-of-plane conductivity of the electrode. Normally for composites, this would be a percolation relation similar to Equation (5). However, here, in the presence of electrolyte, the matrix $\left(\mathrm{Co}(\mathrm{OH})_{2}\right)$ conductivity is much higher than typically found in 
conductor/insulator composites (typically $\sim 10^{-10} \mathrm{~S} \mathrm{~m}^{-1}$ ). This means one cannot neglect parallel conductive paths through the matrix and it is more appropriate to write the out-of-plane electrode conductivity as: $\sigma_{+}=\sigma_{+, \mathrm{Co}(\mathrm{OH}) 2}+\sigma_{+, 0}\left(\phi-\phi_{+, c}\right)^{n_{+}}$, where $\sigma_{+, \mathrm{Co}(\mathrm{OH}) 2}$ is the out-of-plane $\mathrm{Co}(\mathrm{OH})_{2}$ conductivity and $\sigma_{+, 0}, \phi_{+, c}$ and $n_{+}$are the out-of-plane versions of the parameters in Equation (5). This leads to the expression:

$$
\frac{\tau}{C_{A}}=\left(\frac{1}{G_{A}}+\frac{t}{\sigma_{+, \mathrm{Co}(\mathrm{OH}) 2}+\sigma_{+, 0}\left(\phi-\phi_{+, c}\right)^{n_{+}}}\right)
$$

This equation predicts the $\phi$-dependence of $\tau / C_{A}$ once an out-of-plane nanotube network has formed (i.e. $\phi>\phi_{+, c}$ ). We plotted $\tau / C_{A}$ versus $\phi$ in Figure $3 \mathrm{~h}$ (using $\left.C_{A}=\left(C_{M}\right)_{\text {Comp }} \rho_{\text {film }} t\right)$. As in Figure $3 \mathrm{~g}$, the data decays for $\phi>1 \mathrm{vol} \%$. In order to apply Equation (7) in our case, it is necessary to substitute the empirically observed volume fraction dependence of $t$ (see Figure 2a, $\left.t=t_{0}+\left(t_{1}-t_{0}\right) \phi\right)$. Then, taking the empirical values of $t_{0}$ and $t_{1}$ (extracted from data in Figure 3a), and fixing $\phi_{+, c}=1$ vol\% (from Figure 3f) and $\sigma_{+, \mathrm{Co}(\mathrm{OH}) 2}=4.8 \times 10^{-4} \mathrm{~S} \mathrm{~m}^{-1}$ (Figure $2 \mathrm{~h}$ ) we obtained a very good fit using Equation (7). This yielded a value of $\mathrm{G}_{\mathrm{A}}=5430 \pm 460 \mathrm{~S} \mathrm{~m}^{-2}$, consistent with the previous value.

More importantly, the fits yield values of $\mathrm{n}_{+}=2.1 \pm 0.1$ and $\sigma_{+, 0}=4.4 \pm 0.6 \mathrm{~S} \mathrm{~m}^{-1}$ and is consistent with $\phi_{+, c}=1$ vol\%. As far as we are aware, these out-of-plane percolation fit parameters are the first reported for a nano:nano composite. These values are notably different to the in-plane values reported above $\left(\mathrm{n}_{=}=1.45 \pm 0.13, \sigma_{=, 0}=(6 \pm 1) \times 10^{5} \mathrm{~S} \mathrm{~m}^{-1}\right.$ and $\phi_{=, c}$ $=0.25 \pm 0.08$ vol\%). While the nature of the differences is discussed in detail the SI, the most important point is that the out-of-plane conductivity varies much more weakly with $\phi$ compared to the in-plane conductivity. This is largely because of the relatively low value of $\sigma_{+, 0}$ which 
may be due to layering effects and might be increased by improving the electrode deposition method.

This means that the capacitance enhancements on SWNT addition have been achieved with relatively modest out-of-plane conductivity increases. For example, the capacitance saturation at 3 vol\% observed in Figure 3 h occurs at an out-of-plane conductivity of $\sim 2 \times 10^{-3} \mathrm{~S}$ $\mathrm{m}^{-1}$, which is only $\times 4$ times the $\mathrm{Co}(\mathrm{OH})_{2}$ conductivity. In addition, as indicated above, optimizing high scan-rate performance requires $\sigma_{+}>G_{A} t$. Using the fit parameters given above, we find this requires a relative modest conductivity of $\sigma_{+}>6 \times 10^{-3} \mathrm{~S} \mathrm{~m}^{-1}$, which is achieved for $\phi>5.3 \mathrm{vol} \%$. Above this loading, the electrolyte resistance begins to dominate Equation (4), becoming fully rate-limiting for loading levels above $\sim 8$ vol\% as indicated by the saturation of the data in Figure $3 \mathrm{~h}$ at high $\phi$.

At this point, it is worth summarizing the role of conductive additives such as carbon nanotubes in enhancing the performance of low-conductivity electrode materials. This work shows that the nanotubes play two distinct roles related to their conductivity. Firstly, they introduce a conductive network into the electrode which can deliver charge effectively to every part of the electrode. Essentially, this can be thought of as extending the current collector into the interior of the electrode. This allows the electrode material to reach its full potential and increases the intrinsic specific capacity towards the maximum value associated with the accessible surface area of the electrode. Secondly, the nanotubes increase the electrode conductivity, reducing the equivalent series resistance to its thickness-independent minimum (so long as $\sigma_{+}>G_{A} t$ ). This results in minimized charge/discharge rates, significantly increasing capacitance at high rates (Equation (4)).

\section{Thickness dependent performance for $\mathrm{Co}(\mathrm{OH})_{2} / \mathrm{SWNT}$ film electrodes}


In addition to reducing electrical limitations on the capacitance, adding nanotubes improves the mechanical properties of the films allowing thicker electrodes to be fabricated, thus increasing the areal capacitance. However, at some thickness, one would expect the capacitance to become diffusion limited, resulting in reduced applicability of the resistance limited model. To test this, we prepared a series of $\mathrm{Co}(\mathrm{OH})_{2} / \mathrm{SWNT}\left(M_{f}=6 \mathrm{wt} \%\right)$ composite electrodes with thickness ranging from $260 \mathrm{~nm}$ to $11 \mu \mathrm{m}\left(0.1 \mathrm{mg} \mathrm{cm}^{-2} \leq M / A \leq 1.6 \mathrm{mg} \mathrm{cm}^{-2}\right)$. The mass loading $(M / A)$ scaled linearly with thickness, $t$, leading to values of $\left\langle\rho_{\text {film }}\right\rangle=1500 \mathrm{~kg} / \mathrm{m}^{3}$ and $\langle P\rangle \sim 55 \%$. CV measurements were performed as before, with the higher scan-rate data (e.g. $1000 \mathrm{mV} / \mathrm{s}$, Figure 4a) showing the capacitance to fall with increasing thickness consistent with increased charging time. ${ }^{[21]}$

The specific capacitance, is plotted in Figure. $4 \mathrm{~b}$ versus scan-rate and appears similar to the previously discussed data. However, when we attempted to fit the data using equation 1 , we found good fits over the entire scan-rate range only for electrodes with $t<5 \mu \mathrm{m}(M / A \leqslant$ $0.8 \mathrm{mg} \mathrm{cm}^{-2}$ ). For thicker electrodes, clear deviations were observed between data and fit at high rates (Figure 4c). Such deviations are clearly linked to the fact that, at high rate, the thicker films empirically display $C \propto \dot{V}^{-3 / 2}$ (Figure 4c) rather than the $C \propto \dot{V}^{-1}$ behavior expected for resistance limited samples. The breakdown in the model can be seen by plotting values of $\tau / C_{A}$ extracted from the fit versus $M / A$ : clearly the data begins to deviate significantly from the expected behavior (solid line) once $t>3-5 \mu \mathrm{m}$.

We believe that this deviation from purely electrically limited behavior marks the onset of limitations associated with ion diffusion. Such effects are common for thicker electrodes ${ }^{[14}$, ${ }^{32]}$ and are usually consistent with capacitance scaling as $C \propto \dot{V}^{-1 / 2}$ at high rate. However, for thick composite electrodes at high rates, rather than $C \propto \dot{V}^{-1 / 2}$ (diffusion limited) or $C \propto \dot{V}^{-1}$ 
(resistance limited) behavior, we observe $\dot{V}^{-3 / 2}=\dot{V}^{-1} \times \dot{V}^{-1 / 2}$, which may reflect a combination of resistance and diffusion limited behavior.

We found that the capacitance versus rate curves for the thickest composite electrodes, can be well-fitted over the entire scan-rate range using Equation (1), once the time constant is modified to include a term in $\dot{V}^{1 / 2}$ (i.e. $\left.\tau=\tau_{0}+k \dot{V}^{1 / 2}\right)$ :

$$
\frac{C}{M}=\frac{C_{A}}{M / A}\left[1-\frac{\dot{V}\left(\tau_{0}+k \dot{V}^{1 / 2}\right)}{\Delta V}\left(1-e^{-\Delta V / \dot{V}\left(\tau_{0}+k \dot{V}^{1 / 2}\right)}\right)\right]
$$

While we cannot provide a physical justification for this term, it makes intuitive sense that additional rate limiting steps such as diffusion would slow charging more severely at high rates. The composite specific capacitance versus scan-rate data has been reproduced in Figure 4d and fitted to Equation (8), with excellent fits obtained in all cases.

From the fits, we find $C_{A}$ to scale linearly with electrode thickness as expected (Figure 4e), approaching $1 \mathrm{~F} \mathrm{~cm}^{-2}$ for the thickest electrodes. Such values are relatively high compared to literature values (see Table S1 in SI). This data is consistent with intrinsic specific and volumetric capacitances of $490 \mathrm{~F} \mathrm{~g}^{-1}$ and $705 \mathrm{~F} \mathrm{~cm}^{-3}$ respectively. This volumetric capacitance is comparable to the state-of-art $\mathrm{MXene}, \mathrm{MoS}_{2}$ and $\mathrm{MoO}_{3}$ films with similar or thinner thickness (see Table S2 in SI). ${ }^{[33]}$

We have plotted the fit constants, $\tau_{0}$ and $k$, versus electrode loading in Figure $4 \mathrm{f}-\mathrm{g}$. In Figure $4 \mathrm{f}$, the $\tau_{0}$ data for the three thinnest electrodes, almost exactly reproduces the $\tau$ data found using Equation (1) (see SI). We have illustrated this by plotting Equation (2), using parameters given in the panel, obtaining a near perfect match to the data. However, for the four thickest electrodes, we found $\tau_{0} \sim 0$, indicating that, for the thicker films, the electrical resistance-limited mechanism plays a minor role. The second fit constant, $k$, which we suggest represents diffusion limitations, is plotted versus $M / A$ in Figure $4 \mathrm{~g}$. This parameter is extremely 
small for the three thinnest electrodes, but increases significantly for the thicker films. Interestingly, $k$ scales as $(M / A)^{2}$, suggesting it may be related to the time taken by ions to diffuse from the bulk electrolyte all the way into the porous electrode (time to diffuse distance $\mathrm{x}$ scales as $\left.\mathrm{x}^{2}\right)$.

Even with the onset of diffusion limitations, the composite electrodes display high areal capacitances over the whole scan-rate range with performance which is considerably better than $\mathrm{Co}(\mathrm{OH})_{2}$-only films. This is illustrated in Figure $4 \mathrm{~h}$ which compares the best $\mathrm{Co}(\mathrm{OH})_{2}-$ only film with a $6 \mathrm{wt} \%$ composite of equivalent thickness. This shows up to $\times 40$ capacitanceenhancement depending on scan rate. To demonstrate the potential of these high areal capacitances materials, we fabricated $\mathrm{SC}$ devices based on $\mathrm{Co}(\mathrm{OH})_{2} / \mathrm{SWNT}$ films which display energy and power densities as high as $660 \mathrm{~mJ} \mathrm{~cm}^{-2}$ and $103 \mathrm{~mW} \mathrm{~cm}^{-2}$, close to the state of the art (see SI).

\section{CONCLUSIONS}

In this work we have combined a model system based on liquid-exfoliated $\mathrm{Co}(\mathrm{OH})_{2}$ nanosheets with theoretical analysis to elucidate the performance-limiting-parameters in lowconductivity SC electrodes. While in such systems the low-rate areal capacitance can be increased by increasing the electrode thickness, we find that this strategy results in a fall in the high-rate capacitance. This is caused by the increased charging time associated with the scaling of electrode resistance with thickness. The resultant near inverse scaling of high-rate capacitance with electrode thickness will occurs so long as the electrode conductivity is less than the product of the electrolyte areal conductance and the electrode thickness $\left(\sigma_{+}>G_{A} t\right)$.

These problems can be resolved by adding carbon nanotubes to improve the electrode conductivity with the added benefit of mechanically stabilizing the system. The increased conductivity imparted by the nanotubes plays two important roles. Firstly, once $\sigma_{+} \gg G_{A} t$, the 
high-rate capacitance no longer scales with $t$, reaching its maximum value, limited only by the electrolyte conductance. Secondly, nanotubes improve charge distribution, allowing the intrinsic capacitance of the electrode to increase to its surface-area-limited maximum. These effects can yield a $\times 40$ increase in capacity at a given electrode thickness. Our model quantitatively describes these effects, and yields a deep understanding of electrode operation. An example of this can be seen in Figure 5 where we used the models described in this work, as well as the fit parameters obtained, to map out the areal capacitance of a composite electrode as functions of rate, nanotube content and electrode thickness. Such a capability is unprecedented and will allow rational electrode design.

\section{METHODS}

\section{Experimental section}

Preparation of $\mathrm{Co}(\mathrm{OH})_{2}$ dispersions. Liquid cascade centrifugation was used with subsequently increasing rotation speeds as previously reported. $20 \mathrm{~g} \mathrm{~L}^{-1} \mathrm{Co}(\mathrm{OH})_{2}$ in $80 \mathrm{~mL}$ of $9 \mathrm{~g} \mathrm{~L}^{-1}$ sodium cholate was sonicated for $4 \mathrm{~h}$ with a $6 \mathrm{~s}$ on $2 \mathrm{~s}$ off pulse rate. The $80 \mathrm{~mL}$ of sonicated dispersion was centrifuged at $500 \mathrm{rpm}$ (27 g) for $60 \mathrm{~min}$. The sediment was discarded and the supernatant was centrifuged at $1000 \mathrm{rpm}(100 \mathrm{~g})$ for $60 \mathrm{~min}$. The sediment after this centrifugation step was re-dispersed in fresh sodium cholate solution $\left(\mathrm{C}=9 \mathrm{~g} \mathrm{~L}^{-1}, 25 \mathrm{~mL}\right)$ by 5 min bath sonication producing the largest size. The supernatant after the $1000 \mathrm{rpm}$ centrifugation step was centrifuged at $1500 \mathrm{rpm}(240 \mathrm{~g})$ for $60 \mathrm{~min}$, producing the second largest size in the re-dispersed sediment. These steps were repeated in further increments of $2000 \mathrm{rpm}$ (425 g), $2500 \mathrm{rpm}(665 \mathrm{~g})$, and $3000 \mathrm{rpm}$ (950 g). In total five sizes were produced. The smallest sheets were used for SC tests. Chemicals were bought from Sigma-Aldrich.

Preparation of SWNT Dispersions. SWNTs were purchased from Chengdu Organic Chemicals Co. Ltd., China. The SWNTs have a diameter less than $2 \mathrm{~nm}$ and a length ranging 
from 5 to $30 \mu \mathrm{m}$. The SWNTs were used as received. SWNTs were added into sodium cholate solution (with a concentration of $10 \mathrm{mg} \mathrm{mL}^{-1}$ ) at a ratio of $1 \mathrm{mg} \mathrm{SWNT/10} \mathrm{mL} \mathrm{sodium} \mathrm{cholate}$ solution. The dispersion was divided into $14 \mathrm{~mL}$ vials (10 $\mathrm{mL}$ for each vial), and subjected to high power tip sonication for 5 min (25\% amplitude, 2 seconds on and 2 seconds off), then bath sonicated for $30 \mathrm{~min}$ (Branson 1510-MT bath sonication, $20 \mathrm{kHz}, 120 \mathrm{~W}$ ), followed by another $5 \mathrm{~min}$ of tip sonication. The dispersions were centrifuged (5500 rpm for $90 \mathrm{~min}$ ) to get the stable and well-dispersed SWNT supernatant. The concentration of the produced dispersion was measured to be $0.751 \mathrm{mg} \mathrm{mL}^{-1}$.

Film fabrication. Pure $\mathrm{Co}(\mathrm{OH})_{2}$ and SWNT films were prepared via vacuum filtration of $\mathrm{Co}(\mathrm{OH})_{2}$ and SWNT dispersions separately. Mixed cellulose ester filer membranes (MFMillipore membrane, hydrophilic, $47 \mathrm{~mm}$ in diameter) with $25 \mathrm{~nm}$ pore size were used for the filtration. The loading (mass per unit area, M/A) was precisely controlled via filtering specific volumes of a dispersion with known concentrations, resulting in films with different thicknesses. $\mathrm{Co}(\mathrm{OH})_{2} / \mathrm{SWNT}$ composite films were made by filtering a mixture of diluted $\mathrm{Co}(\mathrm{OH})_{2}$ and SWNT dispersions. A sodium cholate aqueous solution $\left(6 \mathrm{mg} \mathrm{mL}^{-1}\right)$ was used to dilute the dispersions to around 10-fold. The mixtures were put into the sonic bath for $30 \mathrm{~min}$ before the filtration. $\mathrm{B}, \mathrm{N}$ co-doped carbon nanosheet/SWNT films were made by filtering a mixture of $\mathrm{B}, \mathrm{N}$ co-doped carbon nanosheet and SWNT dispersions. The mixtures were subjected to sonic bath for $30 \mathrm{~min}$ before filtration.

To remove the sodium cholate as much as possible, all the films were washed via filtering 200 $\mathrm{mL}$ deionized water through the as-made films, and dried overnight at room temperature. After drying, the films were cut into the desired sizes and transferred onto glass substrates or glassy carbon for thickness, electric conductivity measurement, SEM imaging and electrochemical performance tests. The attached cellulose filter membranes on the films were removed by treatment with acetone vapor (for 5 to $10 \mathrm{~s}$ ) and subsequent acetone baths (for $40 \mathrm{~min}$ ). 
Sheets and film characterization. TEM imaging was performing using a JEOL 2100 operated at $200 \mathrm{kV}$. The diluted $\mathrm{Co}(\mathrm{OH})_{2}$ nanosheet dispersion was drop-casted onto holey carbon grids (400 mesh) and the solvent was evaporated before TEM testing. AFM imaging was carried on a Veeco Nanoscope-IIIa from Digital Instruments. E-head in tapping mode was used for all measurements. $\mathrm{Co}(\mathrm{OH})_{2}$ nanosheets dispersions were diluted with deionized water with ratio of $1: 10$ and drop casted $(10 \mu \mathrm{L})$ on preheated $\left(150^{\circ} \mathrm{C}\right) \mathrm{Si} / \mathrm{SiO}_{2}$ wafers $\left(0.25 \mathrm{~cm}^{2}\right)$ with an oxide layer of $300 \mathrm{~nm}$. After deposition wafers were rinsed with deionized water and dried with condensed air prior to measurement. Typical image size taken was $8 \mu \mathrm{m}^{2}$ for overview images and $4 \mu \mathrm{m}^{2}$ for zoomed in images with 512 lines/image and scan rates of $0.8 \mathrm{~Hz}$. Measured thickness was converted to number of layers based on conducted steep height analysis. Film thickness were analyzed using a profilometer (Dektak 6M, Veeco Instruments). Each film was measured at five different locations to get an average thickness. This thickness measurement is non-destructive, allowing the film thickness to be obtained before the conductivity test.

Electrical conductivity was tested via a four-probe method. Silver wire (diameter of $0.5 \mathrm{~mm}$ ) was glued to the film using silver paint (Agar Scientific), the distance between silver wire contacts was carefully measured using ImageJ software. A Keithley 2400 source meter (Keithley Instruments, Inc.) was used to measure the I-V curves.

The morphology of the films was analyzed using a ZEISS Ultra Plus microscopy (Carl Zeiss Group).

Raman spectra were acquired using a Horiba Jobin Yvon LabRam HR800. $632 \mathrm{~nm}$ was chosen as excitation line. Signal was collected using a 100x objective (0.8 N.A.). 600 grooves per mm grating has been chosen in order to obtain $\sim 1.2 \mathrm{~cm}^{-1}$ spectral resolution. Measurements were done in air at room temperature. Beam size on sample was approximately $2 \mu \mathrm{m}$ and the laser power was kept at $0.2 \mathrm{~mW}$. No degradation or heating effects were observed at the used fluence. 
Each plotted spectra is the result of acquiring signal for 60 seconds and the average of 15 spectra is displayed.

Electrochemical performance tests. The SC performance of the as-made films were studied using cyclic voltammetry (CV), galvanostatic charge-discharge (GCD), and electrochemical impedance spectroscopy (EIS). Electrochemical tests were performance using a Reference 3000 Gamry potentiostat. The tests were conducted in a three-electrode system at room temperature, using $\mathrm{Hg} / \mathrm{HgO}$ reference electrode and platinum foil as the counter electrode with $1 \mathrm{M} \mathrm{NaOH}$ electrolyte. The film transferred onto glassy carbon was used as the working electrode. Each electrode was first cycled at $100 \mathrm{mV} \mathrm{s}^{-1}$ for 200 cycles between 0 and $0.6 \mathrm{~V}$ (vs $\mathrm{Hg} / \mathrm{HgO}$ ) to ensure stable charge/discharge behavior. The $\mathrm{CV}$ scan rates ranged from 2 to $5000 \mathrm{mV} \mathrm{s}^{-1}$, the GCD current densities ranged from 3 to $70 \mathrm{~A} \mathrm{~g}^{-1}$. EIS was conducted in the frequency range of 0.1 to $10^{5} \mathrm{~Hz}$ with perturbation voltage amplitude of $10 \mathrm{mV}$.

The electrode thickness, $\mathrm{t}$, is related to the mass loading, M/A, via: $M / A=\rho_{\text {film }} t$, where $\rho_{\text {film }}$ is the electrode density.

The gravimetric capacitance was calculated from the $\mathrm{CV}$ by integrating the current passed during the discharge sweeps: $C_{g}=\frac{1}{M \Delta V \dot{V}} \int_{V}^{V+\Delta V} j d V$

where the $C_{g}$ is the gravimetric capacitance, $M$ is the mass of the electrode, $\Delta V$ is the potential window, $\dot{V}$ is the scan rate, and $j$ is the current density.

The gravimetric capacitance was also calculated from GCD using the following equation: $C_{g}=\left(I_{\text {cons }} / M\right) /(d V / d t)$, where $I_{\text {cons }}$ is the galvanostatic discharge current, $M$ is the electrode mass, and $d V / d t$ is the rate of change of the cell voltage during discharge. 
It is important to clarify the relationship between specific, areal and volumetric capacitances.

For each film we controlled the device area, $A$, and the mass deposited (giving a value for $M$ ) and measured the thickness, $t$. These parameters were used to transform measured capacitance $(C)$ to specific $(C / M)$, areal $(C / A)$, and volumetric $(C / V)$ capacitances as well as electrode density $\left(\rho_{\text {film }}=M / A t\right)$. The following relationships can also be used: $C / V=\rho_{\text {film }} C / M$ (similar for intrinsic values: $C_{V}=C_{M} \rho_{\text {film }}$ ) and $C / A=t \times C / V=C / M \times M / A$, where $M / A$ is the electrode mass loading.

\section{Acknowledgements}

This work was predominantly funded by Science Foundation Ireland (11/PI/1087) as well as the European Union Seventh Framework Programme under grant agreement $n^{\circ} 604391$ Graphene Flagship and the Science Foundation Ireland (SFI) funded centre AMBER (SFI/12/RC/2278).

\section{References}

[1] M. Salanne, B. Rotenberg, K. Naoi, K. Kaneko, P. L. Taberna, C. P. Grey, B. Dunn, P. Simon, Nature Energy, 2016, 1, 16070.

[2] L. Cao, F. Xu, Y. Y. Liang, H. L. Li, Adv. Mater., 2004, 16, 1853; T. Deng, W. Zhang, O. Arcelus, J.-G. Kim, J. Carrasco, S. J. Yoo, W. Zheng, J. Wang, H. Tian, H. Zhang, X. Cui, T. Rojo, Nature Commun., 2017, 8, 15194; P. Simon, Y. Gogotsi, Nat. Mater., 2008, 7, 845.

[3] S. Gao, Y. F. Sun, F. C. Lei, L. Liang, J. W. Liu, W. T. Bi, B. C. Pan, Y. Xie, Angew. Chem.,Int. Ed., 2014, 53, 12789.

[4] K. K. Lee, W. S. Chin, C. H. Sow, J. Mater. Chem. C., 2014, 2, 17212.

[5] B. Marinho, M. Ghislandi, E. Tkalya, C. E. Koning, G. de With, Powder Technol., 2012, 221, 351.

[6] G. Lota, K. Fic, E. Frackowiak, Energy Environ. Sci., 2011, 4, 1592; A. Ansaldo, P. Bondavalli, S. Bellani, A. E. D. Castillo, M. Prato, V. Pellegrini, G. Pognon, F. Bonaccorso, Chemnanomat, 2017, 3, 436.

[7] Y. Liu, X. He, D. Hanlon, A. Harvey, U. Khan, Y. Li, J. N. Coleman, ACS Nano, 2016, 10, 8821.

[8] D. McAteer, Z. Gholamvand, N. McEvoy, A. Harvey, E. O’Malley, G. S. Duesberg, J. N. Coleman, ACS Nano, 2016, 10, 672.

[9] J. N. Coleman, M. Lotya, A. O’Neill, S. D. Bergin, P. J. King, U. Khan, K. Young, A. Gaucher, S. De, R. J. Smith, I. V. Shvets, S. K. Arora, G. Stanton, H.-Y. Kim, K. Lee, G. T. Kim, G. S. Duesberg, T. Hallam, J. J. Boland, J. J. Wang, J. F. Donegan, J. C. Grunlan, G. Moriarty, A. 
Shmeliov, R. J. Nicholls, J. M. Perkins, E. M. Grieveson, K. Theuwissen, D. W. McComb, P. D. Nellist, V. Nicolosi, Science, 2011, 331, 568; R. J. Smith, P. J. King, M. Lotya, C. Wirtz, U. Khan, S. De, A. O'Neill, G. S. Duesberg, J. C. Grunlan, G. Moriarty, J. Chen, J. Wang, A. I. Minett, V.

Nicolosi, J. N. Coleman, Adv. Mater., 2011, 23, 3944.

[10] K. R. Paton, E. Varrla, C. Backes, R. J. Smith, U. Khan, A. O’Neill, C. Boland, M. Lotya, O. M. Istrate, P. King, T. Higgins, S. Barwich, P. May, P. Puczkarski, I. Ahmed, M. Moebius, H. Pettersson, E. Long, J. Coelho, S. E. O’Brien, E. K. McGuire, B. M. Sanchez, G. S. Duesberg, N. McEvoy, T. J. Pennycook, C. Downing, A. Crossley, V. Nicolosi, J. N. Coleman, Nat. Mater., 2014, 13,624 .

[11] C. Backes, B. M. Szydłowska, A. Harvey, S. Yuan, V. Vega-Mayoral, B. R. Davies, P.-1. Zhao, D. Hanlon, E. J. G. Santos, M. I. Katsnelson, W. J. Blau, C. Gadermaier, J. N. Coleman, ACS Nano, 2016, 10, 1589.

[12] Z. P. Liu, R. Z. Ma, M. Osada, K. Takada, T. Sasaki, J. Am. Chem. Soc., 2005, 127, 13869.

[13] H. D. Lutz, H. Möller, M. Schmidt, J. Mol. Struct., 1994, 328, 121; S. R. Shieh, T. S. Duffy, Physical Review B, 2002, 66, 134301.

[14] T. M. Higgins, D. McAteer, J. C. Coelho, B. Mendoza Sanchez, Z. Gholamvand, G. Moriarty, N. McEvoy, N. C. Berner, G. S. Duesberg, V. Nicolosi, J. N. Coleman, ACS Nano, 2014, 8, 9567.

[15] G. Brauer, 1965, 1521.

[16] A. G. Kelly, T. Hallam, C. Backes, A. Harvey, A. S. Esmaeily, I. Godwin, J. Coelho, V. Nicolosi, J. Lauth, A. Kulkarni, S. Kinge, L. D. A. Siebbeles, G. S. Duesberg, J. N. Coleman, Science, 2017, 356, 69.

[17] M. Toupin, T. Brousse, D. Bélanger, Chem. Mater., 2004, 16, 3184.

[18] H. Y. Lee, S. W. Kim, H. Y. Lee, Electrochem. Solid-State Lett., 2001, 4, A19.

[19] A. J. Bard, L. R. Faulkner, John Wiley \& Sons, Inc., 2000.

[20] Y. Wimalasiri, R. Fan, X. S. Zhao, L. Zou, Electrochim. Acta, 2014, 134, 127.

[21] T. M. Higgins, J. N. Coleman, ACS Appl. Mater. Interfaces, 2015, 7, 16495.

[22] D. Hanlon, C. Backes, T. M. Higgins, M. Hughes, A. O’Neill, P. King, N. McEvoy, G. S. Duesberg, B. Mendoza Sanchez, H. Pettersson, V. Nicolosi, J. N. Coleman, Chem. Mater., 2014, 4, 1751.

[23] G. Cunningham, U. Khan, C. Backes, D. Hanlon, D. McCloskey, J. F. Donegan, J. N. Coleman, J. Mater. Chem. C, 2013, 1, 6899; X. Tian, M. E. Itkis, E. B. Bekyarova, R. C. Haddon, Sci. Rep., 2013, 3, 1710.

[24] F. Tronel, L. Guerlou-Demourgues, M. Ménétrier, L. Croguennec, L. Goubault, P. Bernard, C. Delmas, Chem. Mater., 2006, 18, 5840; A. Cressent, V. Pralong, A. Audemer, J.-B. Leriche, A. Delahaye-Vidal, J.-M. Tarascon, Solid State Sci., 2001, 3, 65; F. Lichtenberg, K. Kleinsorgen, J. Power Sources, 1996, 62, 207.

[25] W. Wei, X. Cui, W. Chen, D. G. Ivey, Chem. Soc. Rev., 2011, 40, 1697.

[26] J. Yang, C. Yu, X. Fan, Z. Ling, J. Qiu, Y. Gogotsi, J. Mater. Chem. C., 2013, 1, 1963.

[27] Z. Fan, J. Yan, L. Zhi, Q. Zhang, T. Wei, J. Feng, M. Zhang, W. Qian, F. Wei, Adv. Mater., 2010, 22, 3723; L. Wen, F. Li, H.-M. Cheng, Adv. Mater., 2016, 28, 4306.

[28] G. Cunningham, M. Lotya, N. McEvoy, G. S. Duesberg, P. van der Schoot, J. N. Coleman, Nanoscale, 2012, 4, 6260.

[29] Dietrich Stauffer, A. Aharony, Taylor and Francis, 1994.

[30] W. Bauhofer, J. Z. Kovacs, Compos. Sci. Technol., 2009, 69, 1486.

[31] M. Alhabeb, M. Beidaghi, K. L. Van Aken, B. Dyatkin, Y. Gogotsi, Carbon, 2017, 118, 642.

[32] Y. Xu, Z. Lin, X. Zhong, X. Huang, N. O. Weiss, Y. Huang, X. Duan, Nature Commun.,

2014, 5, 4554; K.-C. Tsay, L. Zhang, J. Zhang, Electrochim. Acta, 2012, 60, 428.

[33] M. Acerce, D. Voiry, M. Chhowalla, Nature Nanotechnology, 2015, 10, 313; M. Ghidiu, M.

R. Lukatskaya, M.-Q. Zhao, Y. Gogotsi, M. W. Barsoum, Nature, 2014, 516, 78; X. Xiao, H. Song, S. Lin, Y. Zhou, X. Zhan, Z. Hu, Q. Zhang, J. Sun, B. Yang, T. Li, L. Jiao, J. Zhou, J. Tang, Y. Gogotsi, Nature Commun., 2016, 7, 11296. 

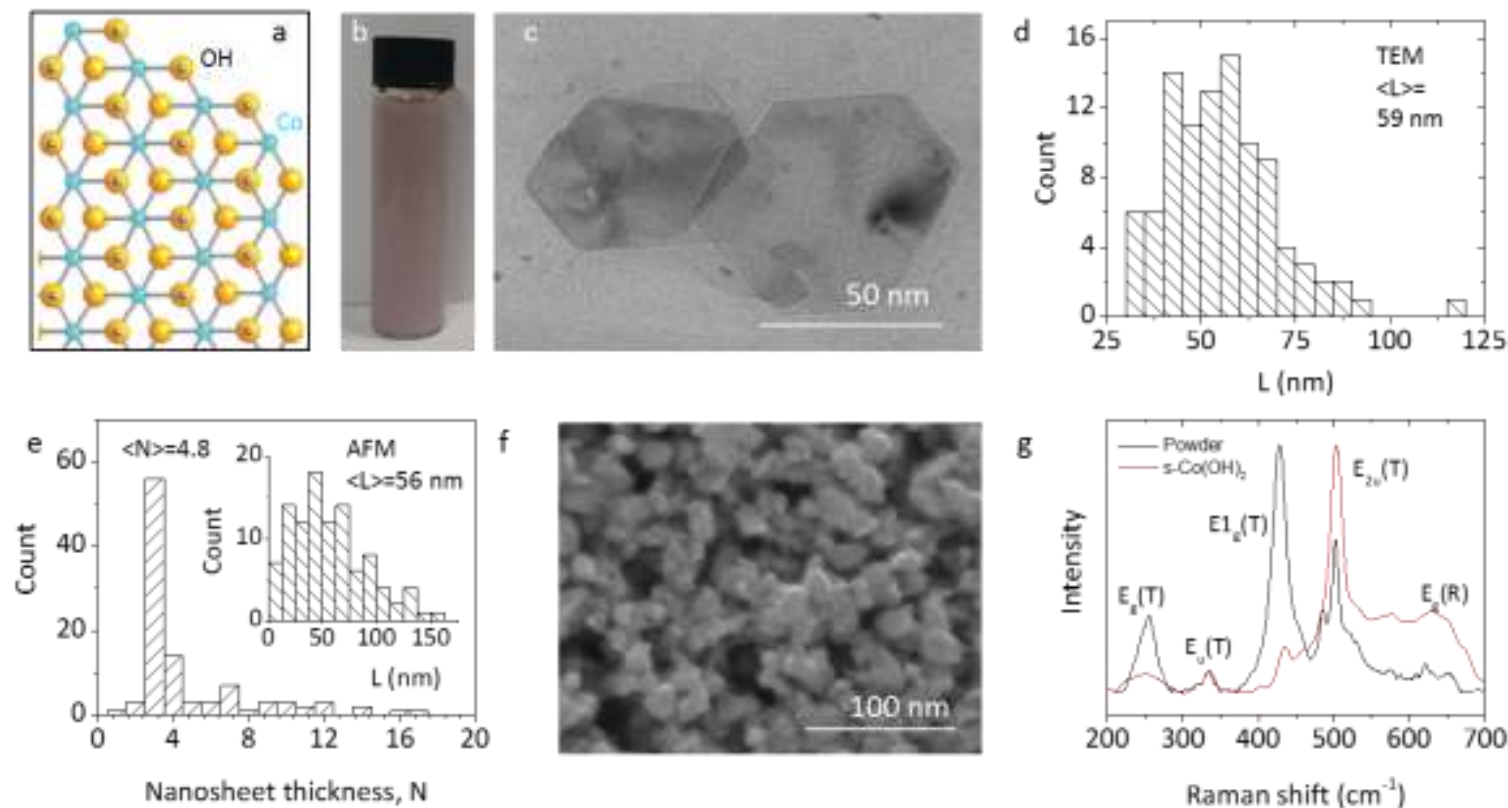

g

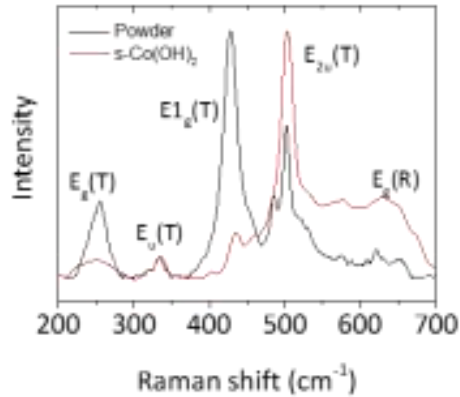

Figure 1. Characterization of liquid exfoliated $\mathrm{Co}(\mathrm{OH})_{2}$ nanosheets. (a) Structure of single layer $\mathrm{Co}(\mathrm{OH})_{2}$. Green, cobalt; yellow, oxygen; gray, hydrogen. (b) Digital image of $\mathrm{Co}(\mathrm{OH})_{2}$ nanosheet dispersion. (c) Typical TEM image of $\mathrm{Co}(\mathrm{OH})_{2}$ nanosheets and (d) TEM-based nanosheet length histogram. (e) AFM-based histogram of $\mathrm{Co}(\mathrm{OH})_{2}$ nanosheet thickness, presented as number of monolayers per nanosheet. Inset: AFM-based nanosheet length histogram. (f) SEM image of a vacuum filtered film of exfoliated $\mathrm{Co}(\mathrm{OH})_{2}$ nanosheets. $(\mathrm{g})$ Raman spectra of original $\mathrm{Co}(\mathrm{OH})_{2}$ powder and the filtered film of exfoliated $\mathrm{Co}(\mathrm{OH})_{2}$ nanosheets. 

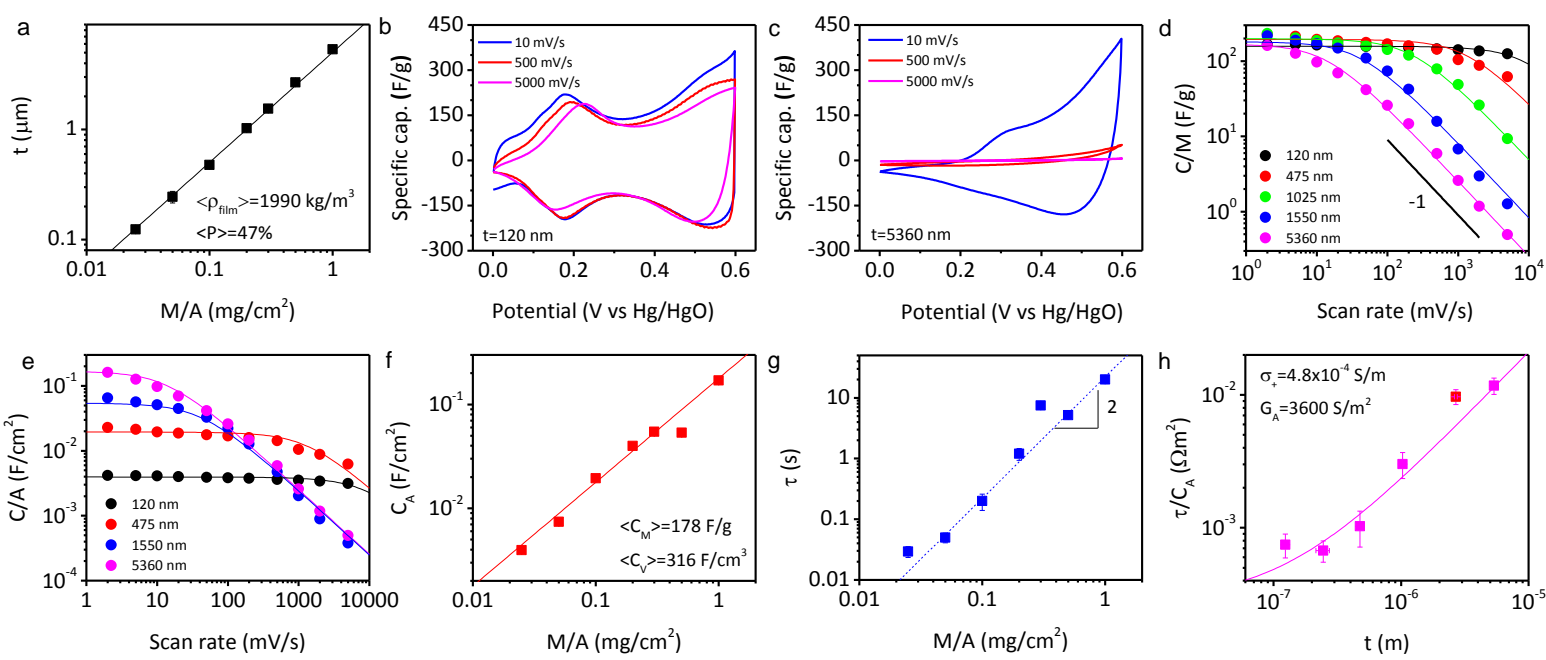

Figure 2. The thickness-dependent supercapacitor performance for electrodes assembled from $\mathrm{Co}(\mathrm{OH})_{2}$ nanosheets. (a) Relationship between mean electrode thickness and mass loading for films of $\mathrm{Co}(\mathrm{OH})_{2}$ nanosheets. (b-c) Cyclic voltammetry curves, measured at various rates, for $\mathrm{Co}(\mathrm{OH})_{2}$ nanosheet electrodes with thickness of 120 (b) and $5360 \mathrm{~nm}$ (c). (d-e) Measured capacitance, normalised to both electrode mass (d) and geometric area (e), plotted versus scanrate for $\mathrm{Co}(\mathrm{OH})_{2}$ electrodes with a range of thickness. In d-e, the lines represent fits to Equation (1). (f-g) Intrinsic areal capacitance (f) and time constant (g), as extracted from the fits in d-e, plotted as function of electrode mass loading. (h) Ratio of time constant to intrinsic areal capacitance plotted versus thickness of $\mathrm{Co}(\mathrm{OH})_{2}$ nanosheet electrodes. The line is a fit to equation 2 . 

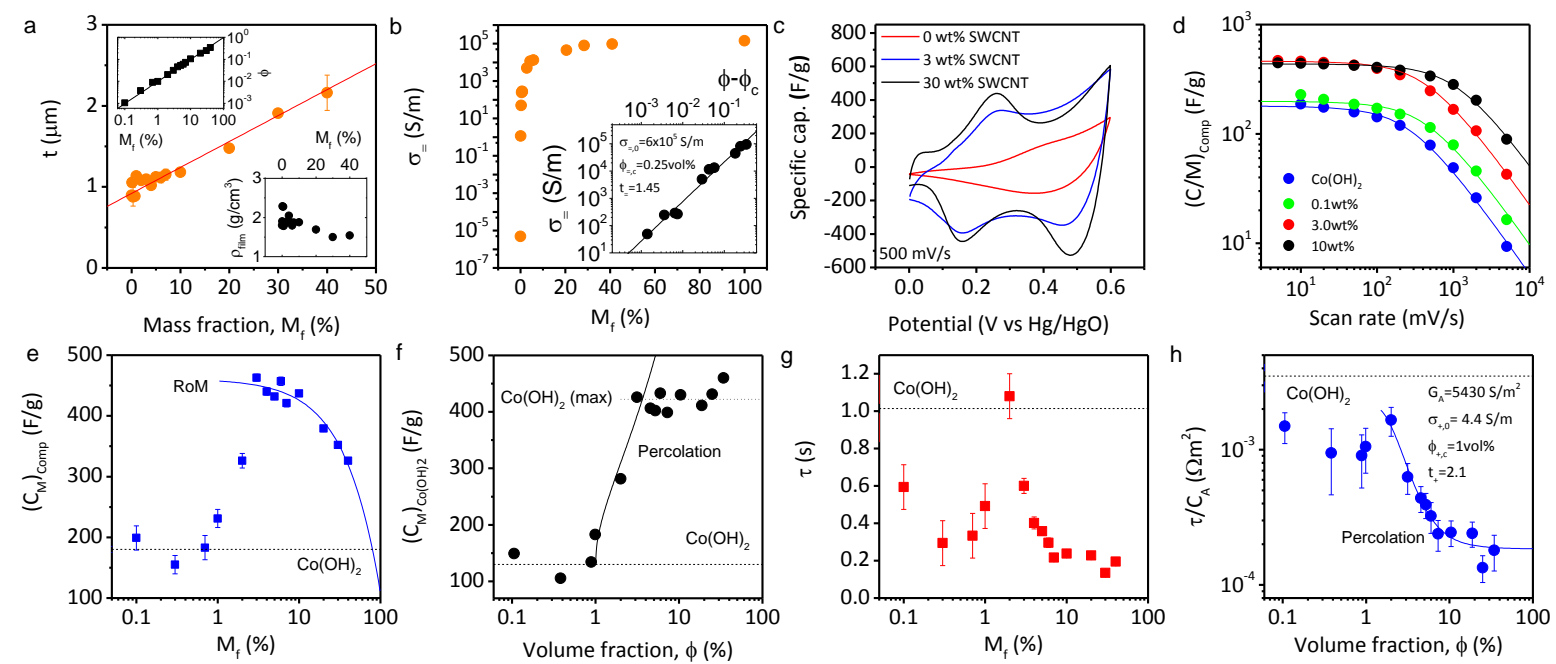

Figure 3. The effect of SWNT content on the performance of $\mathrm{Co}(\mathrm{OH})_{2}$ nanosheet supercapacitor electrodes. (a) Relationship between mean electrode thickness and nanotube mass fraction for $\mathrm{Co}(\mathrm{OH})_{2}$ nanosheets/nanotube films. Insets: Volume fraction (upper) and density (lower) as a function of mass fraction. The solid line in the upper inset represents $\phi=M_{f}$. This data means we can model the electrode thickness using $t=t_{0}+\left(t_{1}-t_{0}\right) \phi$, with $\mathrm{t}_{0}=0.92 \mu \mathrm{m}$ and $\mathrm{t}_{1}-\mathrm{t}_{0}=3.2 \mu \mathrm{m}$. (b) Dry in-plane electrical conductivity of $\mathrm{Co}(\mathrm{OH})_{2}$ nanosheet/nanotube composite films as a function of nanotube mass fraction. Inset: Percolation plot showing in-plane conductivity plotted versus reduced volume fraction, $\phi-\phi_{c}$. The line is a fit to Equation (5). (c) Cyclic voltammograms for $\mathrm{Co}(\mathrm{OH})_{2}$ based film electrodes with 0, 3 , and $30 \mathrm{wt} \%$ SWNTs. (d) Specific capacitance (normalised to total composite electrode mass) of $\mathrm{Co}(\mathrm{OH})_{2} / \mathrm{SWNT}$ composite films as function of scan-rate for a range of SWNT mass fractions. The lines are fits to Equation (1). (e) Intrinsic specific capacitance as extracted from the fits in d, plotted as function of nanotube mass fraction. (f) Intrinsic capacitance of composite electrodes with SWNT contribution removed and normalised to mass of $\mathrm{Co}(\mathrm{OH})_{2}$-only, plotted versus nanotube volume fraction. The upper dotted line represents the maximum achievable specific capacitance of these $\mathrm{Co}(\mathrm{OH})_{2}$ nanosheets. (g) Time constant from fits, plotted as 
function of nanotube mass fraction. (h) Ratio of intrinsic areal capacitance to time constant plotted versus nanotube volume fraction. The solid line is a fit to Equation (7). In e-h, the dashed lines represent the data for a $\mathrm{Co}(\mathrm{OH})_{2}$-only film. All the measured composites had a $\mathrm{Co}(\mathrm{OH})_{2}$ loading of $0.2 \mathrm{mg} \mathrm{cm}^{-2}$.
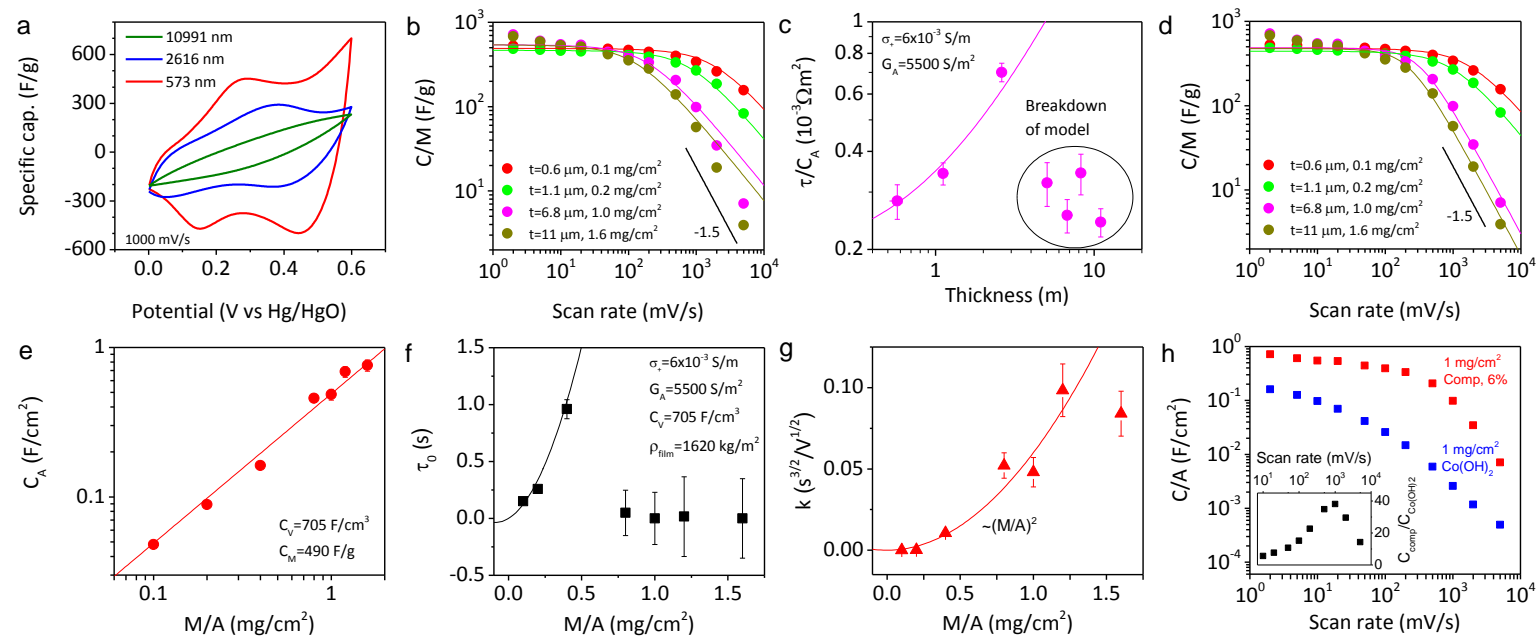

Figure 4. The thickness-dependent supercapacitor performance of $\mathrm{Co}(\mathrm{OH})_{2} / \mathrm{SWNT}$ composite electrodes. (a) Cyclic voltammograms for $\mathrm{Co}(\mathrm{OH})_{2} / \mathrm{SWNT}$ film electrodes with thickness of $573,2616,10991 \mathrm{~nm}$ at $1000 \mathrm{mV} \mathrm{s}^{-1}$. (b) Measured capacitance, normalised to electrode mass plotted versus scan-rate for composite electrodes with a range of thickness. In $b$, the lines represent fits to Equation (1). (c) Ratio of time constant to intrinsic areal capacitance (both from fits to equation 1) plotted versus thickness for composite electrodes. In c, the line represents a plot of Equation (1) using the parameters given in the panel. In addition, the circles enclose those data points representing electrodes which were clearly not solely electrically limited. As such Equation (1) no longer applies. (d) Same data as (b) with the lines representing fits to Equation (8). (e-g) Fit parameters associated with Equation (8). The line in (f) is a plot of Equation (2) using the parameters in the figure and substituting $t$ using $M / A=\rho_{\text {film }} t$. The 
line in (g) represents quadratic behaviour. (h) Comparison of areal capacitance versus rate for the $\mathrm{Co}(\mathrm{OH})_{2}$-only and composite electrodes with $\mathrm{M} / \mathrm{A}=1.0 \mathrm{mg} \mathrm{cm}^{-2}$. Inset: Ratio of composite to $\mathrm{Co}(\mathrm{OH})_{2}$-only areal capacitance. All the tested $\mathrm{Co}(\mathrm{OH})_{2} / \mathrm{SWNT}$ composite films have identical SWNT mass fraction of $6 \mathrm{wt} \%$.
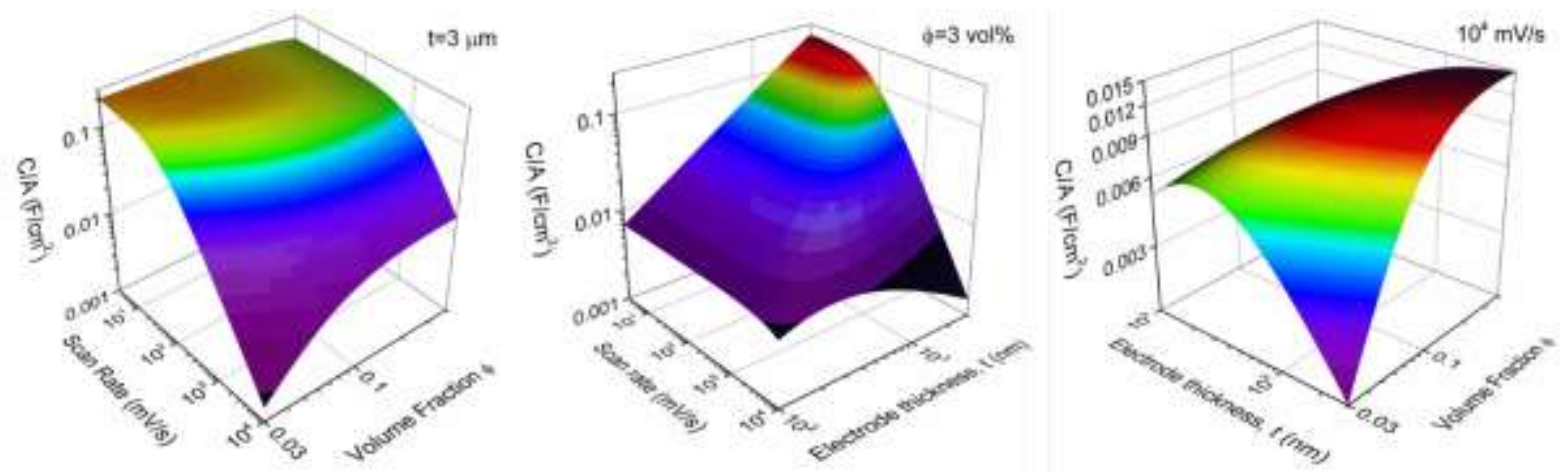

Figure 5. 3D plots of the resistance-limited areal capacitance of $\mathrm{Co}(\mathrm{OH})_{2} / \mathrm{SWNT}$ composite electrodes as a function of combinations of scan rate, volume fraction and electrode thickness. These plots was generated using the models and fit parameters discussed in the text, as described in more detail in the SI, and are calculated each fixing one of the three variables as indicated in the panels. 